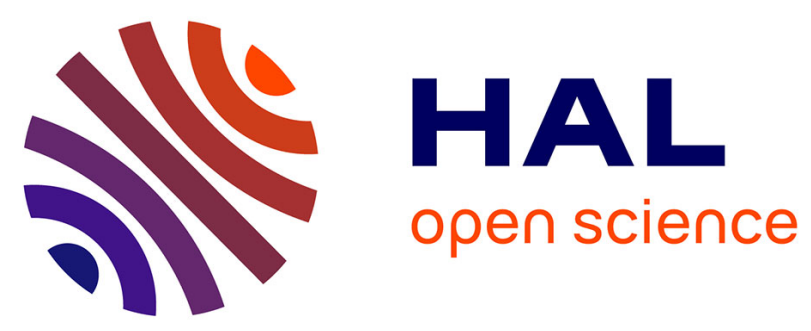

\title{
A Direct Preconditioner for Coupling Matrix Reconfiguration of Bandpass Filters With Irregular Couplings Using Continuation Method
}

\author{
Yan Zhang, Ke-Li Wu, Fabien Seyfert
}

\section{- To cite this version:}

Yan Zhang, Ke-Li Wu, Fabien Seyfert. A Direct Preconditioner for Coupling Matrix Reconfiguration of Bandpass Filters With Irregular Couplings Using Continuation Method. IEEE Transactions on Microwave Theory and Techniques, 2021, 69 (2), pp.1394-1403. 10.1109/TMTT.2020.3045991 . hal03214963

\author{
HAL Id: hal-03214963 \\ https://hal.inria.fr/hal-03214963
}

Submitted on 3 May 2021

HAL is a multi-disciplinary open access archive for the deposit and dissemination of scientific research documents, whether they are published or not. The documents may come from teaching and research institutions in France or abroad, or from public or private research centers.
L'archive ouverte pluridisciplinaire HAL, est destinée au dépôt et à la diffusion de documents scientifiques de niveau recherche, publiés ou non, émanant des établissements d'enseignement et de recherche français ou étrangers, des laboratoires publics ou privés. 


\title{
A Direct Preconditioner for Coupling Matrix Reconfiguration of Bandpass Filters With Irregular Couplings Using Continuation Method
}

\author{
Yan Zhang, Student Member, IEEE, Ke-Li Wu, Fellow, IEEE, and Fabien Seyfert
}

\begin{abstract}
In this paper, a direct preconditioner is proposed for coupling matrix reconfiguration of bandpass filters with irregular couplings using the coefficient-parameter continuation method. With the preconditioner, the continuation method can be effectively used for filter synthesis and model extraction of bandpass filters with irregular couplings. In dealing with practical filters that contain a coupling topology with regular cross couplings introduced intentionally or unintentionally, the proposed preconditioner, being used as the start system in the continuation method, can swiftly lead to the solution that matches the physical realization. The preconditioner is physics-based and can be easily obtained by reconfiguring the regular coupling topology with the conventional method. The continuation process with a coefficient-parameter homotopy for coupling matrix reconfiguration is formulated for the first time, which transforms a coupling matrix in canonical form to that in the desired one with irregular couplings. The convergence of the solution and a bootstrapping approach for dealing with multiple irregular couplings are also discussed. The method provides an enabling tool for robot automatic tuning (RAT) of advanced microwave bandpass filters. Three practical examples are presented, including one synthesis example and two model extraction examples for RAT, demonstrating the effectiveness of the reconfiguration method for practical applications.
\end{abstract}

Index Terms-Coupling matrix, filter reconfiguration, homotopy continuation, irregular topologies, microwave filter tuning, preconditioner.

\section{INTRODUCTION}

$\mathrm{T}$ UNING of high-performance microwave filters is inevitable due to stringent electrical specifications. The labor-intensive manual tuning process, being time-consuming and costly, motivates the recent development of computer-aided tuning (CAT) and robot automatic tuning (RAT), for which coupling matrix [1] is used as the bridge between the physical model and the circuit model of the filter realization in the filter domain. In a robust RAT system, extraction of a legitimate circuit model contains three major

Manuscript received May 7, 2020. The work described in this paper was substantially supported by the Hong Kong Ph.D Fellowship. (Corresponding author: Ke-Li Wu.)

Yan Zhang and $\mathrm{Ke}-\mathrm{Li} \mathrm{Wu}$ are with the Department of Electronic Engineering, The Chinese University of Hong Kong, Shatin, Hong Kong (e-mail: yzhang@link.cuhk.edu.hk; klwu@cuhk.edu.hk). Fabien Seyfert is with INRIA, Sophia-Antipolis, France, (e-mail: fabien.seyfert@inria.fr). steps: 1) to obtain the filtering function characterized by three polynomials from the measured response; 2 ) to transform the filtering function into the circuit model (coupling matrix) in a canonical form; and 3) to reconfigure the coupling matrix in the canonical form to that in the coupling topology that best fits the physical realization. The third step is usually conducted through a sequence of orthogonal transformations. The subsequent tuning strategy of the filter will depend on the difference between the extracted and the target coupling matrices. Among the three steps, the system identification problem in the first step can be addressed using well-developed methods, such as vector fitting [2] and the recently proposed model-based vector fitting (MVF) methods [3]. As long as a set of sensible filtering functions are obtained, a unique coupling matrix in a canonical form can be derived straightforwardly in the second step. However, coupling matrix reconfiguration in the third step using well-established orthogonal transformations [4] is limited to the conventional regular coupling topologies, such as those constructed by cascaded trisections (CTs) and cascaded quartets (CQs) [5].

The demands for dealing with bandpass filters in irregular coupling topologies are frequently encountered in many practical scenarios not only for model extraction but also filter synthesis. Such filters include those with 1) unintentional parasitic cross couplings; 2) intentional but unconventional cross couplings for enhancing the rejection; 3) coupling topologies for evenly distributing power among the internal nodes for high power applications [6]; and 4) a weak dispersion effect, which can be described by parasitic cross couplings.

To reconfigure the coupling matrix of a bandpass filter with an irregular coupling topology, several algorithms are available in the literature. In [7], an exhaustive method is applied to solve a set of polynomial equations by searching for an orthogonal matrix that enforces the non-existing couplings to be zero after similarity transformation. An analytic elimination can be imposed on a set of multiple variable polynomial system to obtain a Gröbner basis for the target coupling topology, with which the orthogonal transformation matrix for obtaining the coupling matrix of an irregular coupling topology can be obtained straightforwardly. Although multiple possible solutions can be found with the Gröbner basis method [8], it is difficult for a RAT application to stick to the target solution that is pertinent to the physical realization.

Some numerical reconfiguration methods using non-linear 
optimization have also been developed. For example, Gauss-Newton method is used in [9] to solve a nonlinear least square problem. A new cost function based on the reflection and transmission coefficients at system zeros and poles of a trial coupling matrix is proposed in [10] and [11], which is minimized to find the coupling matrix with the desired coupling topology. Although these methods have shown some encouraging results for filter synthesis problems, the long computing time and poor convergence to the desired solution make them unfavorable for RAT applications.

To obtain global convergence, the genetic algorithm that is incorporated with local gradient-based optimization is proposed in [12]. The cost function is based on the difference of the polynomial coefficients of the desired and the derived ones from a trial coupling matrix in the desired topology. Multiple possible solutions can be found using randomly generated initial populations in multiple runs. Very recently, an isospectral flow method that avoids direct optimization of the entries in a coupling matrix is proposed [13]. The method gradually adjusts the basis of the vector space pointing to the direction that better fulfills the desired coupling matrix structure. To deal with the multiple solution issue, multiple runs of iterations with random initial values have to be applied exhaustively.

The aforementioned methods have to face the following prohibitive issues for practical use in a RAT process: 1) slow convergence for high order filters; 2) easy to be trapped to a local minimum; and awkwardly, 3) likely falling into an undesired solution. Having said that, this work is the first attempt to overcome these predicaments in the reconfiguration of coupling matrix with an irregular coupling topology.

For RAT applications, it is desirable to extract the coupling matrix from the measured data that 1) matches the physical coupling topology of the filter; 2) is the right solution aligned with the target coupling matrix among multiple possible solutions; and 3) is acquired swiftly to accommodate the real-time tuning. To the best of the authors' knowledge, none of the existing reconfiguration approaches is tailored for these attributes. It is particularly true if a high order bandpass filter with an irregular coupling topology is concerned.

The continuation method first constructs a set of polynomial system with coefficients that are functions of nonzero entries of a canonical coupling matrix, which are considered as parameters. By tracking the change of the unknowns, which are the entries of the orthogonal transformation matrix for reconfiguring the canonical coupling matrix to the one in the wanted coupling topology, with respect to changes in the parameters from the start system, the solution to the current system can be traced efficiently. The proposed algorithm adopts the general concept of the coefficient-parameter homotopy, a mathematic method for finding a specified solution from a known solution (the start system) in a different state. To overcome the difficulty of finding the start system with a solution in a different state of a filter with irregular coupling topology, a new concept called preconditioner is proposed for the first time. The preconditioner is physics-based and can greatly facilitate the continuation process for swiftly finding the coupling matrix that matches the physical realization.

The proposed theory will be validated through three practical examples, including one synthesis example and two model extraction examples for RAT. The first synthesis example is provided for illustrating the basic steps in applying the continuation approach with the proposed preconditioner. The rest of the two tuning examples concern RAT of 10-pole coaxial resonator filters with 6 or $7 \mathrm{TZs}$ for $5 \mathrm{G}$ systems, demonstrating the effectiveness and efficiency of the proposed method for advanced industrial applications. The convergence of the solution and the bootstrapping approach for dealing with multiple irregular couplings are discussed in the examples.

\section{Proposed Reconfiguration Method}

The proposed reconfiguration method starts from establishing a set of polynomial equations according to the coupling topology concerned and the orthogonal transformation matrix to be sought. Unlike the existing numerical methods, whose starting point lacks legitimacy and the convergence is not guaranteed, the proposed method is based on a coefficient-parameter homotopy with a legitimate preconditioner for the start system, leading to fast convergence. The most attractive feature of the proposed method is its inheritability of a solution, which allows the solution at one tuning stage to serve as the start system of the subsequent tuning stage, being perfectly suitable for RAT of bandpass filters.

\section{A. Parameterized Polynomial System}

Following the formulation in [7], $n$ nonzero entries of the orthogonal transformation matrix $\mathbf{Q}$ are set to be the unknown vector $\mathbf{x}=\left[x_{1}, \cdots, x_{n}\right]$. The multi-variable simultaneous polynomial equation system $F(\mathbf{x}, \mathbf{c}(\mathbf{m}))=\mathbf{0}$ is established as

$$
\begin{aligned}
& \mathbf{Q}^{T} \mathbf{Q}-\mathbf{I}=\mathbf{0} \\
& \forall(i, j) \in T,\left(\mathbf{Q}^{T} \mathbf{M}_{C} \mathbf{Q}\right)_{i, j}=0
\end{aligned}
$$

where $\mathbf{M}_{C}$ is a coupling matrix in a canonical form, e.g. the folded form, $T$ is the set of indices of coupling coefficients that are supposed to be zero in the target coupling matrix, and $\mathbf{I}$ is an identity matrix. The coefficient vector $\mathbf{c}$ is composed by the nonzero coupling elements $\mathbf{m}$ in $\mathbf{M}_{C}$, which is considered as the physical parameter vector of the current state. Obviously, the physical parameter vector $\mathbf{m}$ is different for different $\mathbf{M}_{C}$ obtained from different filter responses, which results in different coefficients $\mathbf{c}$ for different polynomial systems. In this regard, the polynomial equation system $F(\mathbf{x}, \mathbf{c}(\mathbf{m}))=\mathbf{0}$ can be used to define the reconfiguration problem for different tuning states of the same filter.

\section{B. Coefficient-Parameter Homotopy Continuation}

The homotopy continuation is a primary computational method used in numerical algebraic geometry, in which a homotopy is formed between any two systems, and the isolated solutions of one are continued to those of the others. According to the polynomial system in (1), the coefficients of the 


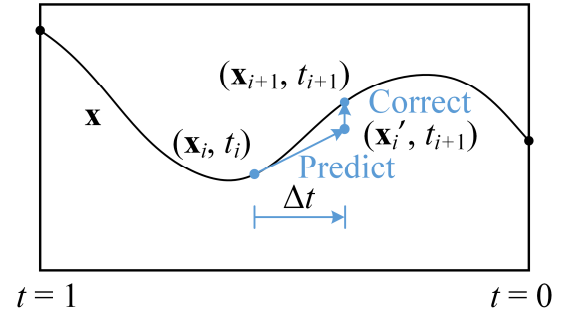

Fig. 1. Path tracking using prediction and correction steps.

TABLE I

PSEUDO-CODE OF THE COEFFICIENT-PARAMETER HOMOTOPY

Input: The polynomial equation system $F(\mathbf{x}, \mathbf{c}(\mathbf{m}))=0$

1) The start system, which is a set of known parameters $\mathbf{m}_{1}$ and the solution $\mathbf{x}_{1}$ for $\mathbf{m}_{1}$.

2) A set of parameters $\mathbf{m}_{0}$ for the system to solve

3) Step size: step

Output: Solution $\mathbf{x}_{0}$ for the system to solve

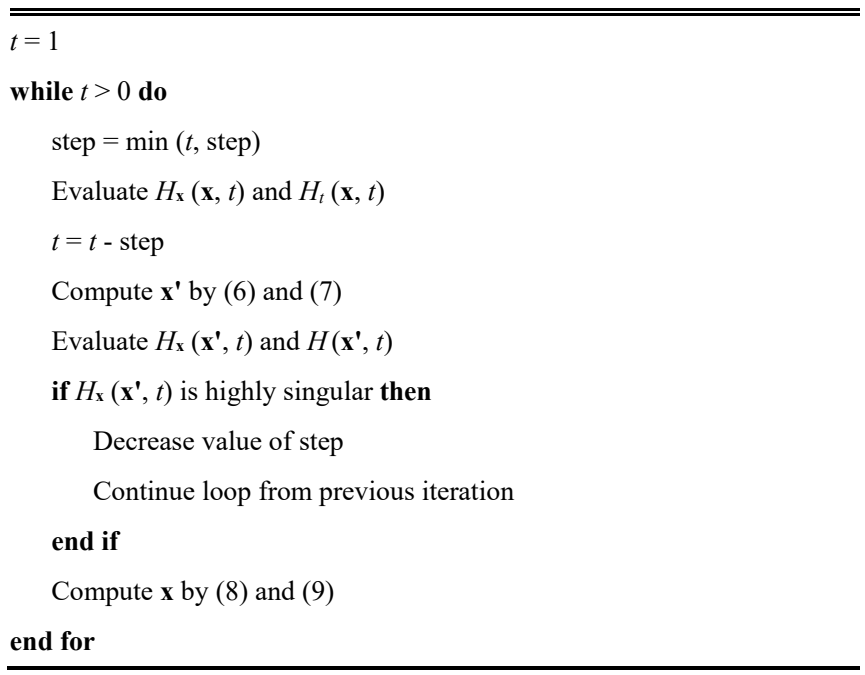

polynomials defined by $\mathbf{c}(\mathbf{m})$ represent a system. Since $\mathbf{c}$ is a continuous function of $\mathbf{m}$, a continuous path through the parameter space leads to a continuous evolution of the coefficients and multiple continuous paths for different solutions. A coefficient-parameter homotopy function can be defined as [14]

$$
H(\mathbf{x}, t)=F\left(\mathbf{x}, \mathbf{c}\left[(1-t) \mathbf{m}_{0}+t \mathbf{m}_{1}\right]\right)
$$

where $\mathbf{m}_{1}$ is the parameter vector corresponding to a known solution with $t=1$, which is called the start system, and $\mathbf{m}_{0}$ is the parameter vector corresponding to the target system with $t=$ 0 . By decreasing $t$ from 1 to 0 , the homotopy function becomes the polynomial system $F\left(\mathbf{x}, \mathbf{c}\left(\mathbf{m}_{0}\right)\right)$, in which $\mathbf{x}$ represents the vector containing the target unknowns to find. Normally, the solution $\mathbf{x}_{1}$ to the start system is supposed to be known beforehand by solving the simultaneous polynomial equations. Usually, such a solution is obtained by an expensive numerical method.

The Euler-Newton path tracking algorithm [15] illustrated in Fig. 1 is applied to track the solution to the polynomial system in (1) from $t=1$ to 0 . Taylor series expansion of the homotopy is applied by

$$
\begin{aligned}
H(\mathbf{x}+\Delta \mathbf{x}, t+\Delta t) & =H(\mathbf{x}, t)+H_{\mathbf{x}}(\mathbf{x}, t) \Delta \mathbf{x}+H_{t}(\mathbf{x}, t) \Delta t \\
& + \text { Higher-Order Terms }
\end{aligned}
$$

where

$$
H_{\mathbf{x}}=\frac{\partial H(\mathbf{x}, t)}{\partial \mathbf{x}}=\frac{\partial F(\mathbf{x}, \mathbf{c})}{\partial \mathbf{x}}
$$

is the Jacobian matrix of $H$ with respect to $\mathbf{x}$ and

$$
H_{t}=\frac{\partial H(\mathbf{x}, t)}{\partial t}=\frac{\partial F(\mathbf{x}, \mathbf{c})}{\partial t}=F\left(\mathbf{x}, \mathbf{c}\left(\mathbf{m}_{1}-\mathbf{m}_{0}\right)\right)
$$

Suppose that the solution $\mathbf{x}_{i}$ to the previous system at $t_{i}$ with respect to $\mathbf{c}_{i}$ is known, or $H\left(\mathbf{x}_{i}, t_{i}\right)=F\left(\mathbf{x}_{i}, \mathbf{c}_{i}\right)=\mathbf{0}$, an approximate solution $\mathbf{x}_{i}^{\prime}$ at $t_{i+1}=t_{i}+\Delta t$ can be predicted by setting $H\left(\mathbf{x}_{i}+\Delta \mathbf{x}\right.$, $\left.t_{i}+\Delta t\right)=0$ so that

$$
\Delta \mathbf{x}=-H_{\mathbf{x}}^{-1}\left(\mathbf{x}_{i}, t_{i}\right) H_{t}\left(\mathbf{x}_{i}, t_{i}\right) \Delta t
$$

and the Euler prediction

$$
\mathbf{x}_{i}^{\prime}=\mathbf{x}_{i}+\Delta \mathbf{x}
$$

Since $H\left(\mathbf{x}_{i}^{\prime}, t_{i+1}\right)$ is not sufficiently small, the solution can be further updated by setting $\Delta t=0$ in (3) at $t_{i+1}$. Then, the prediction (7) can be updated by

$$
\Delta \mathbf{x}=-H_{\mathbf{x}}^{-1}\left(\mathbf{x}_{i}^{\prime}, t_{i+1}\right) H\left(\mathbf{x}_{i}^{\prime}, t_{i+1}\right)
$$

and the Newton correction

$$
\mathbf{x}_{i+1}=\mathbf{x}_{i}^{\prime}+\Delta \mathbf{x}
$$

The pseudocode of the complete continuation process is given in Table I. By gradually changing $t$ from 1 to 0 , the solution corresponding to each $t$ is found using the Euler-Newton method, and the expected solution corresponding to $t=0$ can be eventually sought.

\section{The Preconditioner for Start System}

Suppose the coupling matrix $\mathbf{M}_{1}$ of a response of the filter in the desired topology is known, the start system can be established by transforming $\mathbf{M}_{1}$ to a chosen canonical form $\mathbf{M}_{C 1}$, which can always be done deterministically with transformation matrix $\mathbf{Q}$ defined by

$$
\mathbf{Q M}_{1} \mathbf{Q}^{T}=\mathbf{M}_{C 1}
$$

or

$$
\mathbf{Q}^{T} \mathbf{M}_{C 1} \mathbf{Q}=\mathbf{M}_{1} \quad \text { with } \quad \mathbf{Q}^{T} \mathbf{Q}=\mathbf{I}
$$

in which the nonzero entries in $\mathbf{Q}$ compose solution $\mathbf{x}_{1}$ and the nonzero entries in $\mathbf{M}_{C 1}$ compose $\mathbf{m}_{1}$ such that the start system of $F\left(\mathbf{x}_{1}, \mathbf{c}\left(\mathbf{m}_{1}\right)\right)=0$ is established.

However, for most of the practical cases with irregular coupling topologies, such an $\mathbf{M}_{1}$ is not easy to come by. Although the methods in [7]-[13] can be used to find multiple possible solutions theoretically, the correct solution that matches the filter physical realization can only be picked out by exhaustively examining every solution, which is not practical 


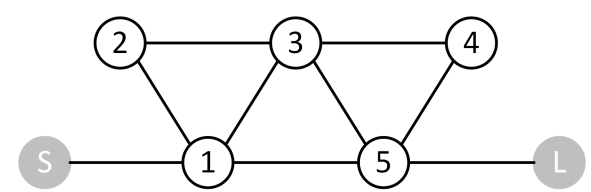

Fig. 2. Coupling topology of a five-pole filter with three TZs.

for RAT. In this work, a physics-based preconditioner for the start system is proposed.

It is very common in practice that an irregular coupling topology composed of a part with regular coupling topology and some additional weak irregular cross couplings. Physically, the filter response is dominated by the regular part whereas the intentionally introduced irregular cross couplings serve as "the icing on the cake". Therefore, the coupling matrix in the regular topology that aligns with the physical solution can be first reconfigured deterministically to construct a preconditioner of the start system. It will be shown through numerical examples that such a preconditioner can very well serve as the start system which effectively leads to the correct solution through the homotopy continuation process. When unintentional irregular cross couplings have to be introduced due to stray couplings, the solution corresponding to the part with regular coupling topology can be obtained by ignoring the far away TZs, leading to a physical preconditioner.

In dealing with an irregular coupling topology with multiple irregular cross couplings, in order to avoid multiple solution problem, a bootstrapping approach can be taken, by which the homotopy continuation process is applied multiple times with a progressive increase of irregular cross couplings. The details will be illustrated in the third example in the next section.

Note that this method is based on the hypothesis that the irregular coupling topology has a real solution for any lossless responses that comply with the shortest path rule [16]. If the irregular cross coupling is poorly located, there is a conceptual possibility that the topology has no physical solution. Nevertheless, an appropriate topology can eventually be obtained by trial-and-error since the failure of convergence of the proposed method indicates the bad choice of topology.

\section{EXAMPLES AND CONVERGENCE OF SOLUTION}

The proposed method is a general scheme not only for circuit model extraction problems but also for direct synthesis problems of bandpass filters with irregular topologies. The first illustrative example will be a synthesis problem of a 5-pole filter with an intentional irregular cross coupling, through which every detail of establishing a homotopy and the continuation process will be given. The second example is a practical 10-pole filter in a regular topology yet with an extra parasitic cross coupling, with which the accuracy of the method is demonstrated. The last example demonstrates how the proposed method is used in a RAT process of a 10-pole filter with more than one irregular couplings, in which the bootstrapping approach is applied.

\section{A. Synthesis of a Five-Pole Filter with Three TZs}

The synthesis of a five-pole filter that generates 3 TZs in the topology depicted in Fig. 2 is considered. Two of the three TZs are placed at $-1.6 j$ and $2.4 j$ in the normalized lowpass frequency domain, which can be easily realized by two CTs. It's convenient to add a cross coupling between resonators 1 and 5 to create the third $\mathrm{TZ}$ at $-8 j$ to enhance the rejection in the far lower rejection band. For such a coupling topology there is no known rotation recipe for reconfiguration.

With the given TZs and specified $20 \mathrm{~dB}$ return loss level, the coupling matrix in the folded canonical form can be easily obtained and is denoted as $\mathbf{M}_{C}$. The first step to reconfigure $\mathbf{M}_{C}$ to the coupling matrix, say $\mathbf{M}_{0}$, in the desired topology is to establish a polynomial equation system. Basically, the orthogonal transformation matrix $\mathbf{Q}$ can be set as a blockwise diagonal matrix diag $(1, \mathbf{Q x}, 1)$ with $\mathbf{Q x}$ being an $N \times N$ block matrix whose elements are unknown [7]. However, the set of the unknowns can usually be reduced to a less-redundant one by reducing the dimension of $\mathbf{Q x}$ such that $\mathbf{Q}=\operatorname{diag}(\mathbf{I}, \mathbf{Q x}, \mathbf{I})$ in a trial-and-error way with symbolic calculation. In this example, the orthogonal transformation matrix $\mathbf{Q}$ can be set in the form of

$$
\mathbf{Q}=\left[\begin{array}{ccccccc}
1 & 0 & 0 & 0 & 0 & 0 & 0 \\
0 & 1 & 0 & 0 & 0 & 0 & 0 \\
0 & 0 & x_{1} & x_{2} & x_{3} & 0 & 0 \\
0 & 0 & x_{4} & x_{5} & x_{6} & 0 & 0 \\
0 & 0 & x_{7} & x_{8} & x_{9} & 0 & 0 \\
0 & 0 & 0 & 0 & 0 & 1 & 0 \\
0 & 0 & 0 & 0 & 0 & 0 & 1
\end{array}\right]
$$

where the unknown vector is $\mathbf{x}=\left[x_{1}, x_{2}, \ldots, x_{9}\right]$. Comparing $\mathbf{Q}^{T} \mathbf{M}_{C} \mathbf{Q}$ against the desired coupling topology, the homotopy function $H$ is formulated according to (2) as

$$
H=\left\{\begin{array}{l}
a_{3} x_{3}=0 \\
a_{4} x_{1} x_{3}+a_{5} x_{3} x_{4}+a_{12} x_{3} x_{7}+a_{5} x_{1} x_{6}+a_{6} x_{4} x_{6} \\
+a_{7} x_{6} x_{7}+a_{7} x_{4} x_{9}+a_{12} x_{1} x_{9}+a_{8} x_{7} x_{9}=0 \\
a_{13} x_{1}+a_{9} x_{7}=0 \\
x_{1} x_{2}+x_{4} x_{5}+x_{7} x_{8}=0 \\
x_{1} x_{3}+x_{4} x_{6}+x_{7} x_{9}=0 \\
x_{2} x_{3}+x_{5} x_{6}+x_{8} x_{9}=0 \\
x_{1}^{2}+x_{4}^{2}+x_{7}^{2}-1=0 \\
x_{2}^{2}+x_{5}^{2}+x_{8}^{2}-1=0 \\
x_{3}^{2}+x_{6}^{2}+x_{9}^{2}-1=0
\end{array}\right.
$$

with $\mathbf{a}=t \mathbf{m}_{1}+(1-t) \mathbf{m}_{0}$ and $\mathbf{m}=\left[M_{s, 1}, M_{1,1}, M_{1,2}, M_{2,2}, M_{2,3}\right.$, $\left.M_{3,3}, M_{3,4}, M_{4,4}, M_{4,5}, M_{5,5}, M_{5, L}, M_{2,4}, M_{2,5}, M_{1,5}\right]$. Subsequently, the Jacobian matrices with respect to $\mathbf{x}$ and $t$ can be found according to (4) and (5).

Assume the TZs $2.4 \mathrm{j}$ and $-1.6 \mathrm{j}$ are assigned to CT (1-2-3) and CT (3-4-5), respectively. Then a physics-based approximation for $\mathbf{M}_{1}$ is chosen to be the coupling matrix in the coupling topology without cross coupling $M_{1,5}$, which can be synthesized as 


$$
\mathbf{M}_{1}=\left[\begin{array}{ccccccc}
0 & 1.0076 & 0 & 0 & 0 & 0 & 0 \\
1.0076 & -0.0105 & 0.8186 & 0.2407 & 0 & 0 & 0 \\
0 & 0.8186 & -0.3641 & 0.5986 & 0 & 0 & 0 \\
0 & 0.2407 & 0.5986 & -0.0288 & 0.5411 & -0.3899 & 0 \\
0 & 0 & 0 & 0.5411 & 0.5466 & 0.7590 & 0 \\
0 & 0 & 0 & -0.3899 & 0.7590 & -0.0105 & 1.0076 \\
0 & 0 & 0 & 0 & 0 & 1.0076 & 0
\end{array}\right]
$$

By transforming $\mathbf{M}_{1}$ to the folded form by a deterministic process as

$$
\mathbf{M}_{C 1}=\left[\begin{array}{ccccccc}
0 & 1.0076 & 0 & 0 & 0 & 0 & 0 \\
1.0076 & -0.0105 & 0.8533 & 0 & 0 & 0 & 0 \\
0 & 0.8533 & -0.0134 & 0.6004 & -0.1257 & -0.1100 & 0 \\
0 & 0 & 0.6004 & 0.2132 & 0.6834 & 0 & 0 \\
0 & 0 & -0.1257 & 0.6834 & -0.0461 & 0.8462 & 0 \\
0 & 0 & -0.1100 & 0 & 0.8462 & -0.0105 & 1.0076 \\
0 & 0 & 0 & 0 & 0 & 1.0076 & 0
\end{array}\right]
$$

one can find the preconditioner $\mathbf{Q}$ for the start system, or

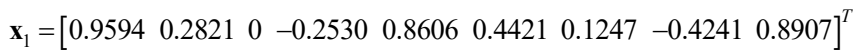

with parameter $\mathbf{m}_{1}$ composed by coupling coefficients in $\mathbf{M}_{C 1}$.

Note that this is a degenerate system since it satisfies the polynomial equation system without cross coupling $M_{1,5}$. The filter response of $\mathbf{M}_{1}$ is shown in Fig. 3 in solid lines. The coupling matrix $\mathbf{M}_{C 0}$ for the system with cross coupling $M_{1,5}$ in folded form can be easily found by the traditional synthesis method as

$\mathbf{M}_{C 0}=\left[\begin{array}{ccccccc}0 & 1.0079 & 0 & 0 & 0 & 0 & 0 \\ 1.0079 & -0.0139 & 0.8539 & 0 & 0 & 0.0118 & 0 \\ 0 & 0.8539 & -0.0159 & 0.5959 & -0.1798 & -0.0961 & 0 \\ 0 & 0 & 0.5959 & 0.2956 & 0.6627 & 0 & 0 \\ 0 & 0 & -0.1798 & 0.6627 & -0.0566 & 0.8484 & 0 \\ 0 & 0.0118 & -0.0961 & 0 & 0.8484 & -0.0139 & 1.0079 \\ 0 & 0 & 0 & 0 & 0 & 1.0079 & 0\end{array}\right]$

and consequently, the parameter $\mathbf{m}_{0}$, which represents the target system to solve.

With the homotopy function and the preconditioner $\mathbf{x}_{1}$ for the start system, the solution for the target system $\mathbf{m}_{0}$ can be found by the coefficient-parameter homotopy continuation method as $\mathbf{x}_{0}=\left[\begin{array}{lllllllll}0.9726 & 0.2325 & 0 & -0.2047 & 0.8563 & 0.4741 & 0.1102 & -0.4611 & 0.8805\end{array}\right]^{T}$ with which the synthesized coupling matrix $\mathbf{M}_{0}$ is found as

$$
\mathbf{M}_{0}=\left[\begin{array}{ccccccc}
0 & 1.0079 & 0 & 0 & 0 & 0 & 0 \\
1.0076 & -0.0139 & 0.8305 & 0.1985 & 0 & 0.0118 & 0 \\
0 & 0.8305 & -0.3074 & 0.6134 & 0 & 0 & 0 \\
0 & 0.1985 & 0.6134 & -0.0453 & 0.5262 & -0.4136 & 0 \\
0 & 0 & 0 & 0.5262 & 0.5759 & 0.7470 & 0 \\
0 & 0.0118 & 0 & -0.4136 & 0.7470 & -0.0139 & 1.0079 \\
0 & 0 & 0 & 0 & 0 & 1.0079 & 0
\end{array}\right]
$$

whose filter response is shown in Fig. 3 in dashed lines. As expected, higher rejection in the lower rejection band is obtained. By perturbing $M_{2,2}$ and $M_{4,4}$ in $\mathbf{M}_{0}$ separately, it is confirmed that TZs corresponding to CT (1-2-3) and CT (3-4-5) are the same as those initially assigned, validating that $\mathbf{M}_{0}$ is the correct solution.

It is interesting to look at the preconditioner from multiple solution point of view. By using a tool like Dedale-HF [8] one can compute all solutions associated to the irregular topology in Fig. 2. It is found that the total number (not counting the classical sign changes), which is the reduced order of the

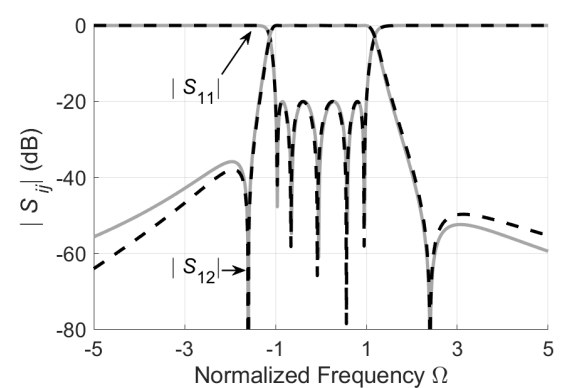

Fig. 3. Response of the start system $\mathbf{M}_{1}$ (solid lines) and the target system $\mathbf{M}_{0}$ (dashed lines) of the 5-pole filter.

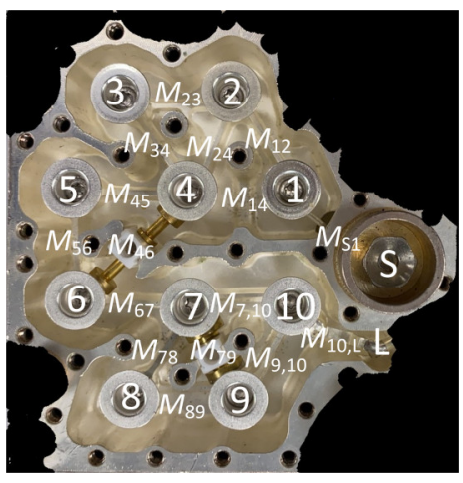

Fig. 4. A clipped photo of the coaxial combline filter (for example B) in a mult-filter module for $5 \mathrm{G}$ applications.

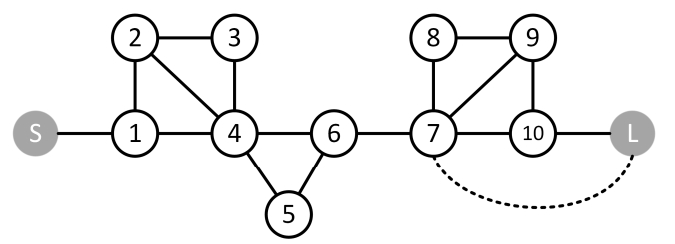

Fig. 5. Topology of the ten-pole filter in Fig. 4 with one CT, two CQs, and a parasitic coupling represented in the dashed line.

topology [7], of solutions in this example is 2 . When the third transmission zero at $-8 \mathrm{j}$ is progressively sent to infinity, the two solutions converge to the two solutions corresponding to the two possible assignments of TZs to the two triplets. This explanation justifies the correspondence between the physics-based preconditioner in a regular coupling topology and the final solution in an irregular coupling topology. In other words, provided that the third TZ is sufficiently far away from the passband, the knowledge of the regular part of the coupling matrix can be used as a preconditioner to uniquely determine the final coupling matrix via the continuation process.

\section{B. A 10-Pole Filter in Regular Topology with a Parasitic TZ}

This example intends to demonstrate the effectiveness of the method in extracting the coupling matrix from the measured filter response. A coaxial combline filter in a multi-filter module that consists of 16 similar filters for a $5 \mathrm{G}$ base station product is shown in Fig. 4, the coupling topology of which shown in Fig. 5 contains one CT and two CQs in its physical realization. A parasitic coupling that is supposedly caused by the stray coupling between resonator 7 and the load, or $M_{7, L}$, is also presented in Fig. 5 in a dashed line. This parasitic coupling is chosen based on the shortest path rule [16] to accommodate 
TABLE II

COUPLing MATRIX $\mathbf{M}_{C 0}$ OF EXAMPLE B

\begin{tabular}{|c|c|c|c|c|c|}
\hline$M_{S 1}$ & $M_{12}$ & $M_{23}$ & $M_{34}$ & $M_{45}$ & $M_{56}$ \\
\hline $\begin{array}{c}1.0406 \\
-0.0029 j\end{array}$ & $\begin{array}{c}0.7662 \\
-0.0005 j\end{array}$ & $\begin{array}{c}0.6148 \\
+0.0002 \mathrm{j}\end{array}$ & $\begin{array}{c}0.5440 \\
-0.0004 j\end{array}$ & 0.3990 & $\begin{array}{c}0.5681 \\
-0.0004 j\end{array}$ \\
\hline$M_{67}$ & $M_{78}$ & $M_{89}$ & $M_{9,10}$ & $M_{10, L}$ & $M_{57}$ \\
\hline $\begin{array}{r}0.1916 \\
+0.0005 \mathrm{j} \\
\end{array}$ & $\begin{array}{c}0.5050 \\
-0.0008 \mathrm{j} \\
\end{array}$ & $\begin{array}{r}0.7218 \\
+0.0001 \mathrm{j} \\
\end{array}$ & $\begin{array}{c}0.8559 \\
-0.0003 \mathrm{j} \\
\end{array}$ & $\begin{array}{c}1.0426 \\
-0.0018 \mathrm{j} \\
\end{array}$ & $\begin{array}{r}0.0566 \\
+0.0008 \mathrm{j} \\
\end{array}$ \\
\hline$M_{47}$ & $M_{48}$ & $M_{38}$ & $M_{39}$ & $M_{29}$ & \\
\hline-0.5232 & $\begin{array}{c}0.1237 \\
+0.0008 \mathrm{j} \\
\end{array}$ & 0 . & $\begin{array}{c}-0.0312 \\
-0.0003 \mathrm{j} \\
\end{array}$ & $\begin{array}{r}-0.0009 \\
+0.0001 \mathrm{j} \\
\end{array}$ & \\
\hline$M_{S S}$ & $M_{11}$ & $M_{22}$ & $M_{33}$ & $M_{44}$ & $M_{55}$ \\
\hline $\begin{array}{c}-0.0016 \\
-0.0142 j\end{array}$ & $\begin{array}{c}0.4309 \\
-0.0134 j\end{array}$ & $\begin{array}{l}-0.5044 \\
-0.0073 \mathrm{j}\end{array}$ & $\begin{array}{c}-0.2215 \\
-0.0069 j\end{array}$ & $\begin{array}{c}-0.4212 \\
-0.0075 \mathrm{j}\end{array}$ & $\begin{array}{l}-0.1097 \\
-0.0073 \mathrm{j}\end{array}$ \\
\hline$M_{66}$ & $M_{77}$ & $M_{88}$ & $M_{99}$ & $M_{10,10}$ & $M_{L L}$ \\
\hline $\begin{array}{c}0.6160 \\
-0.0082 \mathrm{j}\end{array}$ & $\begin{array}{c}0.1665 \\
-0.0061 \mathrm{j}\end{array}$ & $\begin{array}{l}-0.0512 \\
-0.0068 j\end{array}$ & $\begin{array}{c}0.0358 \\
-0.0074 j\end{array}$ & $\begin{array}{c}0.0152 \\
-0.0196 j\end{array}$ & $\begin{array}{c}0.0043 \\
-0.0218 j\end{array}$ \\
\hline
\end{tabular}

TABLE III

COUPLing MATRIX $\mathbf{M}_{0}$ OF EXAMPLe B

\begin{tabular}{|cccccc|}
\hline$M_{S 1}$ & $M_{12}$ & $M_{14}$ & $M_{23}$ & $M_{24}$ & $M_{34}$ \\
1.0406 & 0.6601 & 0.3889 & 0.1533 & 0.4080 & 0.2240 \\
$-0.0029 \mathrm{j}$ & $-0.0009 \mathrm{j}$ & $+0.0005 \mathrm{j}$ & $+0.0002 \mathrm{j}$ & $-0.0007 \mathrm{j}$ & \\
\hline$M_{45}$ & $M_{46}$ & $M_{56}$ & $M_{67}$ & $M_{78}$ & $M_{79}$ \\
0.5381 & -0.1633 & 0.4533 & 0.5410 & 0.2607 & -0.4610 \\
$+0.0002 \mathrm{j}$ & $+0.0005 \mathrm{j}$ & $-0.0001 \mathrm{j}$ & $-0.0006 \mathrm{j}$ & $-0.0015 \mathrm{j}$ & $+0.0024 \mathrm{j}$ \\
\hline$M_{7,10}$ & $M_{7 L}$ & $M_{89}$ & $M_{9,10}$ & $M_{10, L}$ & \\
0.3834 & 0.0170 & 0.1785 & 0.7751 & 1.0425 & \\
$+0.0027 \mathrm{j}$ & $-0.0011 \mathrm{j}$ & $-0.0016 \mathrm{j}$ & $-0.0024 \mathrm{j}$ & $-0.0018 \mathrm{j}$ & \\
\hline$M_{S S}$ & $M_{11}$ & $M_{22}$ & $M_{33}$ & $M_{44}$ & $M_{55}$ \\
-0.0016 & 0.4309 & -1.0311 & -0.3959 & -0.3722 & 0.1547 \\
$-0.0142 \mathrm{j}$ & $-0.0134 \mathrm{j}$ & $-0.0082 \mathrm{j}$ & $-0.0069 \mathrm{j}$ & $-0.0069 \mathrm{j}$ & $-0.0083 \mathrm{j}$ \\
\hline$M_{66}$ & $M_{77}$ & $M_{88}$ & $M_{99}$ & $M_{10,10}$ & $M_{L L}$ \\
-0.0757 & -0.2720 & 0.9430 & 0.5717 & 0.0028 & 0.0043 \\
$-0.0081 \mathrm{j}$ & $-0.0104 \mathrm{j}$ & $-0.0066 \mathrm{j}$ & $-0.0028 \mathrm{j}$ & $-0.0189 \mathrm{j}$ & $-0.0218 \mathrm{j}$ \\
\hline
\end{tabular}

the parasitic TZ mathematically. The center frequency of the filter $f_{0}=2.591 \mathrm{GHz}$ and the bandwidth $\mathrm{BW}=194 \mathrm{MHz}$.

In this example, the polynomials for the filtering function are identified from the measured S-parameters using MVF with an extra TZ. With the polynomials, $\mathbf{M}_{C 0}$ in the folded form can be easily obtained and is listed in Table II, with which the required polynomial homotopy system can be built according to (1).

It can be easily found by perturbation that the desired $\mathrm{TZ}$ at $-1.865 \mathrm{j}$ is realized by CT $(4-5-6)$; TZ pair at $(1.136 \mathrm{j},+1.237 \mathrm{j})$ are controlled by CQ (1-2-3-4); and TZ pair at (-1.108j, $-1.261 \mathrm{j})$ are controlled by CQ (7-8-9-10). The coupling matrix $\mathbf{M}_{1}$ in coupling topology without $M_{7, L}$ can be easily synthesized with $20 \mathrm{~dB}$ return loss in the specified passband. The solution $\mathbf{x}_{1}$ for the start system can be obtained by transforming $\mathbf{M}_{1}$ to $\mathbf{M}_{\mathrm{C} 1}$, which is in canonical form according to (10). Note that such a start system is a degenerate system since $\mathbf{M}_{1}$ is not exactly in the desired coupling topology. However, the degenerate system can be found efficiently and can serve as a very good preconditioner.

The coupling matrix $\mathbf{M}_{C 0}$ in canonical form is used as the parameters for the target system in the desired topology. By following the procedure given in Table I and using the preconditioner as the start system, the target system $\mathbf{M}_{0}$ in the
TABLE IV

ROTATION SEQUENCE TO RECONFIGURE THE COUPLING MATRIX

\begin{tabular}{|ccc|}
\hline $\begin{array}{c}\text { Rotation } \\
\text { sequence }\end{array}$ & $\begin{array}{c}\text { Elements to be } \\
\text { annihilated }\end{array}$ & $\begin{array}{c}\text { Pivot } \\
{[i, j]}\end{array}$ \\
\hline \hline 1 & {$[8, \mathrm{~L}]$} & {$[8,9]$} \\
2 & {$[9, \mathrm{~L}]$} & {$[9,10]$} \\
3 & {$[8,10]$} & {$[8,9]$} \\
\hline
\end{tabular}

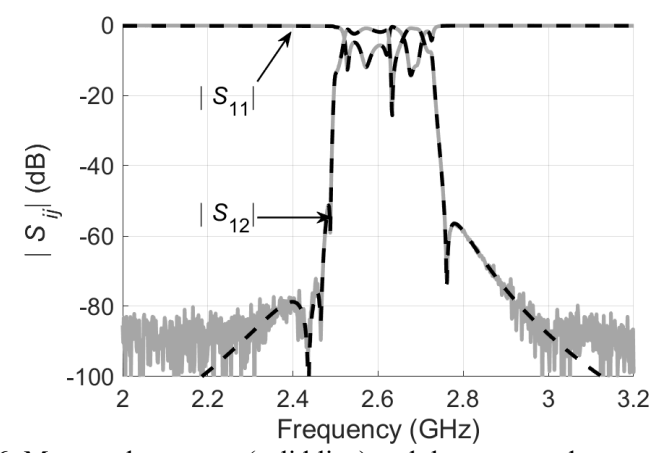

Fig. 6. Measured response (solid line) and the recovered response (dashed line) corresponding to $\mathbf{M}_{0}$ without the imaginary parts of inter-resonator couplings.

coupling topology of Fig. 5 can be obtained as listed in Table III, in which the imaginary part of the self-coupling terms represents the loss. Due to numerical errors in MVF caused by measurement noise and non-physical locations of spurious couplings to a certain extent, a very small amount of imaginary parts may occur in off-diagonal terms. Such small imaginary parts can be omitted without visibly affecting the response. As presented in Fig. 6, the filter response obtained by the reconfigured $\mathbf{M}_{0}$ with the imaginary parts of inter-resonator couplings neglected matches the measured response very well.

In fact, a direct annihilating rotation sequence can be found for this special irregular coupling topology. After the conventional reconfiguration process for obtaining CQ (1-2-3-4) and CT (4-5-6), the final coupling matrix can be reconfigured by 3 more sequentially rotations, as listed in Table IV, for annihilating $M_{8, L}, M_{9, L}$, and $M_{8,10}$, resulting in exactly the same coupling matrix as $\mathbf{M}_{0}$ obtained by the homotopy continuation method. This fact further verifies the proposed preconditioner and the numerical continuation process.

Actually, the existence of coupling $M_{7, L}$ changes the CQ closest to the load port to a quintet in arrow form, which is also a canonical block with a unique solution. Therefore, the degenerate system $\mathbf{M}_{1}$ will evolve to the unique solution if the assignment of TZs to each cascaded block in $\mathbf{M}_{1}$ is the same as that of $\mathbf{M}_{0}$. In fact, there is a one-to-one mapping in solutions between the irregular topology and any other topologies with which the missing cross coupling down the minimum path causes one TZ to vanish consequently. For RAT applications, this feature means that if a regular part of the coupling topology can be clearly identified the coupling matrix for the irregular topology will converge to the unique solution with the preconditioner.

The parasitic coupling $M_{7, L}$ is artificially introduced to the circuit to accommodate the unintentional TZ. It's observed that the coupling value is relatively small and stable during the tuning process. A new golden template matrix is subsequently 


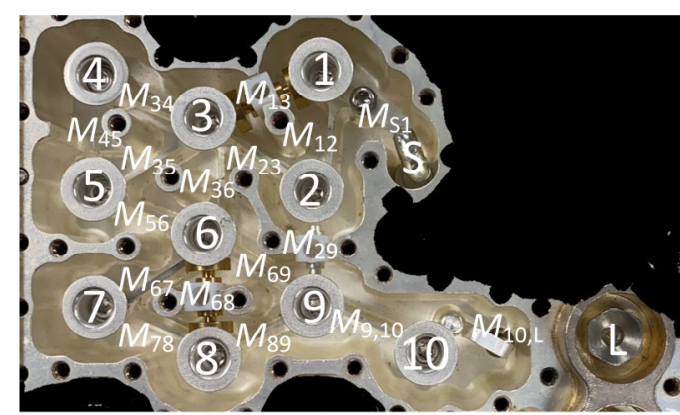

Fig. 7. A clipped photo of the coaxial combline filter (for example C) in a multi-filter module for $5 \mathrm{G}$ applications.

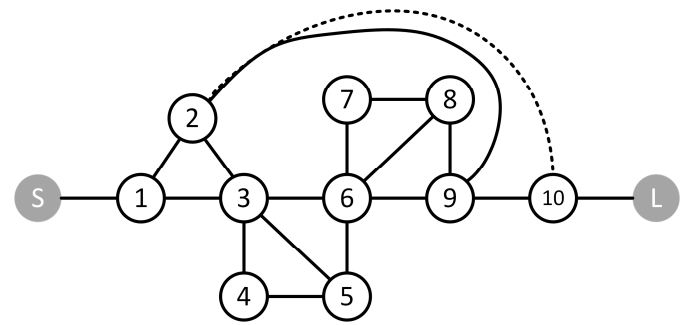

Fig. 8. Topology of a ten-pole filter in Fig. 7 with an irregular cross coupling and a parasitic coupling represented in the dashed line.

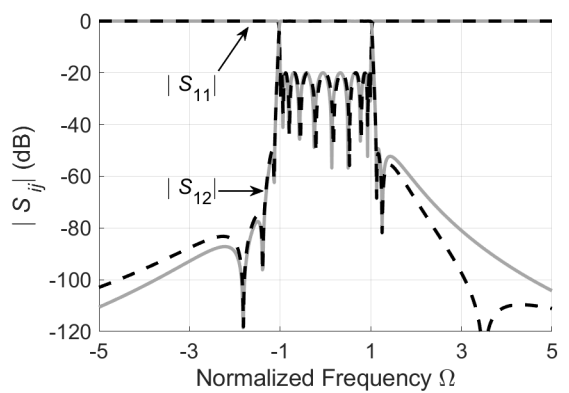

Fig. 9. Response of the preconditioner (solid lines) corresponding to $\mathbf{M}_{1}$ and the synthesized golden template (dashed lines) corresponding to $\mathbf{M}_{0}$ of example C.

generated by incorporating the parasitic TZ. Then, the tuning process can be properly guided with the updated target coupling matrix.

\section{A 10-Pole Filter with both Intentional and Unintentional Irregular Couplings}

Another 10-pole filter is studied in this example, whose physical layout is shown in Fig. 7. This example will illustrate that the coefficient-parameter homotopy continuation can be efficiently applied to model extraction of a high order filter which is with both intentional and unintentional irregular couplings.

As illustrated in Fig. 8, the coupling topology consists of an intentional irregular cross coupling $M_{2,9}$ on top of the regular one CT and two CQs topology, as well as a parasitic coupling $M_{2,10}$, which needs to be introduced to accommodate a parasitic TZ. Coupling $M_{2,9}$ is introduced conveniently to create an extra $\mathrm{TZ}$ to further suppress the rejection since resonators 2 and 9 are physically adjacent.

The extraction process will take two steps in a bootstrapping manner:

Step 1: a golden template coupling matrix that corresponds to the physical layout without considering the parasitic coupling $M_{2,10}$, is synthesized using the proposed preconditioner and the continuation method. Assigning a TZ at $-1.816 \mathrm{j}$ to $\mathrm{CT}(1-2-3)$, a pair of TZs (1.128j, 1.253j) to CQ(3-4-5-6), and a pair of TZs (-1.134j, -1.385j) to CQ(6-7-8-9), the preconditioner $\mathbf{M}_{1}$ for synthesizing the template can be obtained based on the regular part of the filter layout without $M_{2,9}$, and $M_{2,10}$ of course. The coupling matrix $\mathbf{M}_{C 0}$ in folded form for the system with the additional TZ at about $3.5 \mathrm{j}$ due to cross coupling $M_{2,9}$ can be easily found by the traditional synthesis method. The synthesized response is equal-ripple in the passband. Similar to example $\mathrm{A}$ in this Section, the template coupling matrix $\mathbf{M}_{0}$ corresponding to the physical layout can be obtained using the coefficient-parameter homotopy continuation, which is listed in Table V. The responses of the golden template matrix $\mathbf{M}_{0}$ (in dashed lines) is compared to that of the preconditioner of $\mathbf{M}_{1}$ (in solid lines) in Fig. 9. It is observed that with $M_{2,9}$ introduced, the suppression in the higher rejection band is increased.

Step 2: To accommodate the parasitic coupling $M_{2,10}$, which is identified from the parasitic TZ in the measured response of the real filter, the golden template matrix $\mathbf{M}_{0}$ is used as the preconditioner for reconfiguring the final coupling matrix from the measured data. Obviously, the final coupling matrix involves $M_{2,9}$ and $M_{2,10}$. Since the additional TZ introduced by $M_{2,10}$ on top of those of the preconditioner is far away from the passband, applying the homotopy continuation process will lead to a unique solution. The bootstrapping approach can be applied to other reconfiguration problems, in which more than one irregular couplings exist.

TABLE V

COUPling Matrix $\mathbf{M}_{0}$ FOR THE GOLden Template Matrix OF EXAmple C

\begin{tabular}{c|c|c|c|c|c|c|c|c|c|c|c|c|c|c|}
\multicolumn{1}{c|}{} & \multicolumn{1}{c|}{$\mathrm{S}$} & 1 & 2 & 3 & 4 & 5 & 6 & 7 & 8 & 9 & 10 & $\mathrm{~L}$ \\
\hline $\mathrm{S}$ & 0 & 0.9809 & 0 & 0 & 0 & 0 & 0 & 0 & 0 & 0 & 0 & 0 \\
\hline 1 & 0.9809 & 0 & 0.7999 & -0.0937 & 0 & 0 & 0 & 0 & 0 & 0 & 0 & 0 \\
\hline 2 & 0 & 0.7999 & 0.1344 & 0.5723 & 0 & 0 & 0 & 0 & 0 & -0.0034 & 0 & 0 \\
\hline 3 & 0 & -0.0937 & 0.5723 & -0.0179 & 0.2317 & 0.4392 & 0.2075 & 0 & 0 & 0 & 0 & 0 \\
\hline 4 & 0 & 0 & 0 & 0.2317 & -0.9201 & 0.1449 & 0 & 0 & 0 & 0 & 0 & 0 \\
\hline 5 & 0 & 0 & 0 & 0.4392 & 0.1449 & -0.3598 & 0.4843 & 0 & 0 & 0 & 0 & 0 \\
\hline 6 & 0 & 0 & 0 & 0.2075 & 0 & 0.4843 & -0.018 & 0.2376 & -0.4298 & 0.217 & 0 & 0 \\
\hline 7 & 0 & 0 & 0 & 0 & 0 & 0 & 0.2376 & 0.9064 & 0.1669 & 0 & 0 & 0 \\
\hline 8 & 0 & 0 & 0 & 0 & 0 & 0 & -0.4298 & 0.1669 & 0.3512 & 0.5355 & 0 & 0 \\
\hline 9 & 0 & 0 & -0.0034 & 0 & 0 & 0 & 0.217 & 0 & 0.5355 & 0 & 0.8053 & 0 \\
\hline 10 & 0 & 0 & 0 & 0 & 0 & 0 & 0 & 0 & 0 & 0.8053 & 0 & 0.9809 \\
\hline $\mathrm{L}$ & 0 & 0 & 0 & 0 & 0 & 0 & 0 & 0 & 0 & 0 & 0.9809 & 0 \\
\hline
\end{tabular}




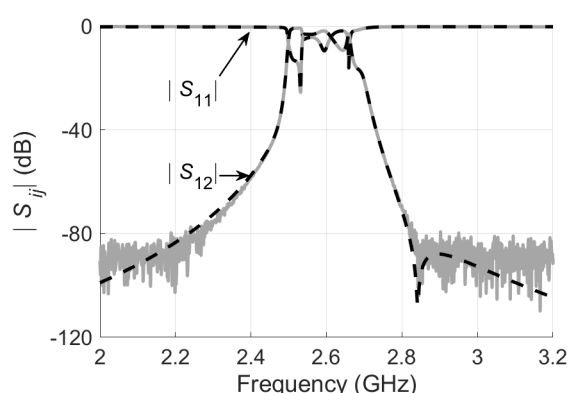

(a)

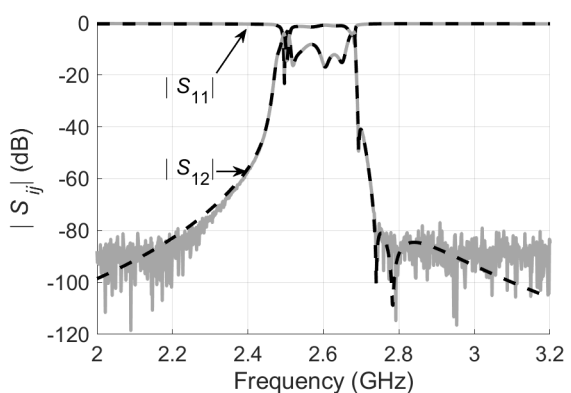

(b)

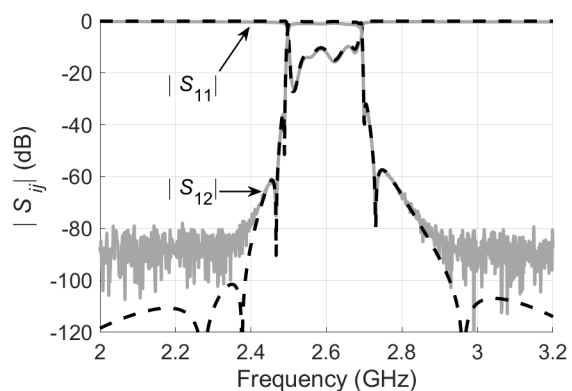

(c)

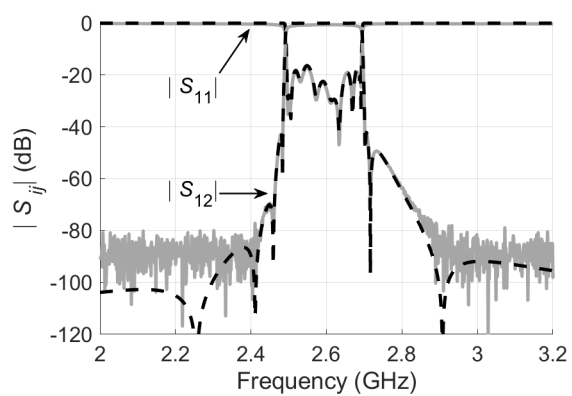

(d)

Fig. 10. Measured data in solid lines and responses by MVF in dashed lines of four tuning stages.

Step 2 can be applied to different tuning stages of a RAT process. Responses of reconfigured coupling matrices and the measured data of four typical tuning stages of the filter are superimposed in Figs. 10(a) - 10(d), showing excellent pertinence of the extracted coupling matrix in each stage. Note that the golden template matrix needs to be constantly updated by taking into account the parasitic coupling in the tuning process.

It is this proposed reconfiguration method that enables a progressively automatic tuning process in a deterministic fashion. The reconfigured coupling matrices in the four tuning stages are listed in Table VI. The elapsed time for reconfiguration of each tuning stage using the proposed method
TABLE VI

Real Parts of Coupling Matrices of the Four Tuning Stages in FIGS. 10 (A)-(D)

\begin{tabular}{|c|c|c|c|c|}
\cline { 2 - 5 } \multicolumn{1}{c|}{} & Stage 1 & Stage 2 & Stage 3 & Stage 4 \\
\hline$M_{S S}$ & -0.0009 & -0.0014 & -0.0012 & -0.0014 \\
\hline$M_{11}$ & -0.0259 & -0.0219 & 0.0017 & 0.0171 \\
\hline$M_{22}$ & -0.0341 & 0.2967 & 0.2768 & 0.3683 \\
\hline$M_{33}$ & 0.1567 & 0.1242 & -0.0526 & -0.0420 \\
\hline$M_{44}$ & -0.6493 & -0.8493 & -0.9499 & -0.9607 \\
\hline$M_{55}$ & 0.1318 & -0.2775 & -0.4011 & -0.4142 \\
\hline$M_{66}$ & -0.2617 & 0.0865 & -0.0210 & -0.0134 \\
\hline$M_{77}$ & 0.5715 & 0.8695 & 0.9461 & 0.9337 \\
\hline$M_{88}$ & 0.3370 & 0.6802 & 0.4027 & 0.3700 \\
\hline$M_{99}$ & 0.0136 & 0.0120 & 0.0135 & 0.0108 \\
\hline$M_{10,10}$ & 0.0492 & 0.0547 & 0.0044 & 0.0204 \\
\hline$M_{L L}$ & 0.0025 & 0.0071 & -0.0074 & 0.0132 \\
\hline$M_{S 1}$ & 1.0594 & 1.0592 & 1.0589 & 1.0586 \\
\hline$M_{12}$ & 0.7882 & 0.8326 & 0.8431 & 0.8430 \\
\hline$M_{23}$ & 0.5091 & 0.5034 & 0.5410 & 0.5740 \\
\hline$M_{34}$ & 0.2235 & 0.2187 & 0.2149 & 0.2165 \\
\hline$M_{45}$ & 0.1680 & 0.1654 & 0.1652 & 0.1265 \\
\hline$M_{56}$ & 0.3889 & 0.4249 & 0.4253 & 0.4854 \\
\hline$M_{67}$ & 0.1913 & 0.1833 & 0.1953 & 0.2387 \\
\hline$M_{78}$ & 0.1760 & 0.1802 & 0.1693 & 0.1677 \\
\hline$M_{89}$ & 0.5581 & 0.5708 & 0.5538 & 0.5537 \\
\hline$M_{9,10}$ & 0.8971 & 0.8967 & 0.8980 & 0.8564 \\
\hline$M_{10, L}$ & 1.0069 & 1.0068 & 1.0047 & 1.0167 \\
\hline$M_{13}$ & -0.2369 & -0.2362 & -0.2043 & -0.2209 \\
\hline$M_{35}$ & 0.4497 & 0.4525 & 0.4559 & 0.4523 \\
\hline$M_{36}$ & 0.1859 & 0.1958 & 0.1833 & 0.2304 \\
\hline$M_{68}$ & -0.4780 & -0.4787 & -0.4708 & -0.4533 \\
\hline$M_{69}$ & 0.2580 & 0.2431 & 0.2717 & 0.2330 \\
\hline$M_{29}$ & -0.0116 & -0.0107 & -0.0027 & -0.0035 \\
\hline$M_{2,10}$ & -0.0007 & -0.0007 & 0.0009 & 0.0009 \\
\hline & & & & \\
\hline
\end{tabular}

with tens of iterations is about 0.75 seconds on average by a MATLAB [17] code on a PC computer.

\section{CONCLUSION}

In this paper, an effective and efficient numerical approach for coupling matrix reconfiguration is proposed by introducing a legitimate and physics-based preconditioner in the parameter-coefficient continuation process. The preconditioner can greatly facilitate the continuation process to most of the practical filter problems, which involve irregular coupling topologies. By introducing the preconditioner, which can be easily obtained using classical filter synthesis theory, for the start system, the continuation process will lead to the unique solution corresponding to the given physical realization. The proposed approach is particularly useful not only for robot automatic tuning (RAT) but also for synthesizing advanced bandpass filters with irregular couplings, which are introduced either intentionally or unintentionally. The effectiveness and efficiency of the proposed approach have been validated and demonstrated through three practical examples, including a synthesis example, an extraction example, and a RAT of a 10-pole filter with $7 \mathrm{TZs}$. It is amazingly seen that the preconditioner can serve as a start system of the continuation 
process very robustly. It is believed that the approach can play an important role in future RAT of advanced microwave bandpass filters.

\section{REFERENCES}

[1] R. J. Cameron, "General coupling matrix synthesis methods for Chebyshev filtering functions," IEEE Trans. Microw. Theory Tech., vol. 47, no. 4, pp. 433-442, Apr. 1999.

[2] $\mathrm{H}$. $\mathrm{Hu}$ and $\mathrm{K} . \mathrm{-L}$. $\mathrm{Wu}$, "A generalized coupling matrix extraction technique for bandpass filters with uneven-Qs," IEEE Trans. Microw. Theory Techn., vol. 62, no. 2, pp. 244-251, Feb. 2014.

[3] P. Zhao and K.-L. Wu, "Model-based vector-fitting method for circuit model extraction of coupled-resonator diplexers," IEEE Trans. Microw. Theory Techn., vol. 64, no. 6, pp. 1787-1797, Jun. 2016.

[4] R. J. Cameron, C. M. Kudsia, and R. Mansour, Microwave Filters for Communication Systems: Fundamentals Design and Applications, 2nd ed. Hoboken, NJ, USA: Wiley, 2018.

[5] S. Tamiazzo and G. Macchiarella,"An analytical technique for the synthesis of cascaded N-tuplets cross-coupled resonators microwave filters using matrix rotations," IEEE Trans. Microw. Theory Tech., vol. 53, no. 5, pp. 1693-1698, May 2005.

[6] B. S. Senior, I. C. Hunter, V. Postoyalko, R. Parry, "Optimum network topologies for high power microwave filters", 6th High Freq. Postgraduate Student Colloq., 2001, pp. 53-58.

[7] R. J. Cameron, J. -C. Faugere, F. Rouillier, and F. Seyfert, "An exhaustive approach to the coupling matrix synthesis problem application to the design of high degree asymmetric filters," Int. J. RF, Microw. Computer-Aided Eng., vol. 17, no. 1, pp. 4-12, Jan. 2007.

[8] F. Seyfert, Dedale-HF. INRIA, Sophia-Antipolis, France, 2006. [Online]. Available: www-sop.inria.fr/apics/Dedale/.

[9] G. Macchiarella, "A powerful tool for the synthesis of prototype filters with arbitrary topology," in IEEE MTT-S Int. Microw. Symp. Dig., July 2003, vol. 3, pp. 1467-1470.

[10] W. Atia, K. Zaki, and A. Atia, "Synthesis of general topology multiple coupled resonator filters by optimization," in IEEE MTT-S Int. Microw. Symp. Dig., Jun. 1998, vol. 2, pp. 821-824.

[11] S. Amari, "Synthesis of cross-coupled resonator filters using an analytical gradient-based optimization technique," IEEE Trans. Microw. Theory Techn., vol. 48, no. 9, pp. 1559-1564, Sep. 2000.

[12] M. Uhm, S. Nam, and J. Kim, "Synthesis of resonator filters with arbitrary topology using hybrid method," IEEE Trans. Microw. Theory Techn., vol.55, no.10, pp. 2157-2167, Oct. 2007.

[13] S. Pflüger, C. Waldschmidt and V. Ziegler, "Coupling matrix extraction and reconfiguration using a generalized isospectral flow method," IEEE Trans. Microw. Theory Techn., vol. 64, no. 1, pp. 148-157, Jan. 2016.

[14] A. J, Sommese and " "Coefficient-parameter polynomial continuation," Appl. Math. Comput., vol. 29, pp. 123-160, Jan. 1989.

[15] A. J. Sommese and C. W. Wampler, The Numerical Solution of Systems of Polynomials Arising in Engineering and Science, Singapore: World Scientific. 2005.

[16] S. Amari, "On the maximum number of finite transmission zeros of coupled resonator filters with a given topology," in IEEE Microw. Guided Wave Lett., vol. 9, no. 9, pp. 354-356, Sep. 1999.

[17] MATLAB, (R2019a), The Mathworks, Natick, Massachusetts, USA.

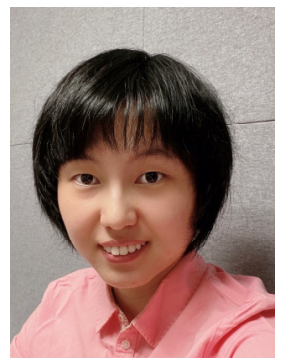

Yan Zhang (S'18) received the B.S. degree in electronic engineering from the University of Electronic Science and Technology of China, Chengdu, China, in 2017. She is currently pursuing the Ph.D. degree at the Chinese University of Hong Kong, Shatin, Hong Kong. Her current research interests include synthesis and tuning of filters with dispersive couplings and filters with irregular topologies.

Ms. Zhang is a recipient of Hong Kong Ph.D. Fellowship.

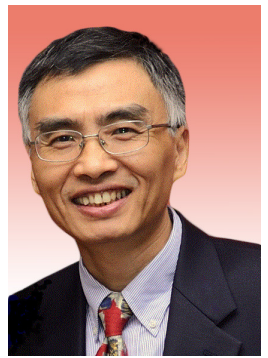

Ke-Li Wu (M'90-SM'96-F'11) received the B.S. and M.Eng. degrees from the Nanjing University of Science and Technology, Nanjing, China, in 1982 and 1985, respectively, and the Ph.D. degree from Laval University, Quebec, QC, Canada, in 1989. From 1989 to 1993, he was a Research Engineer with McMaster University, Canada. He joined COM DEV (now Honeywell Aerospace), Cambridge, ON Canada in 1993, where he was a Principal Member of Technical Staff. Since 1999, he has been with The Chinese University of Hong Kong, Hong Kong, where he is currently a Professor and the Director of the Radiofrequency Radiation Research Laboratory. His current research interests include EM-based circuit domain modeling of high-speed interconnections, robot automatic tuning of microwave filters, decoupling techniques of MIMO antennas, and IoT technologies. Prof. Wu is a member of the IEEE MTT-8 Subcommittee. He was a recipient of the 1998 COM DEV Achievement Award and the Asia-Pacific Microwave Conference Prize twice in 2008 and 2012, respectively. He was an Associate Editor of the IEEE Transactions on MTT from 2006 to 2009 .

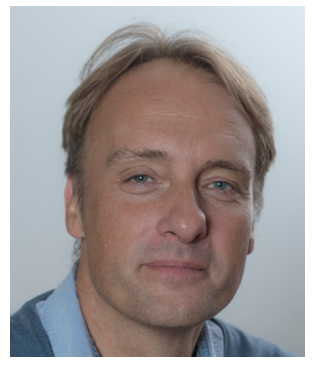

Fabien Seyfert graduated from the "Ecole superieure des Mines" (Engineering School) in St Etienne (France) in 1993 and received his Ph.D. in mathematics in 1998. From 1998 to 2001 he joined Siemens (Munich, Germany) as a researcher specialized in discrete and continuous optimization methods. Since 2002 he occupies a full research position at INRIA (french agency for computer science and control, Nice, France). His research interest focuses on the conception of effective mathematical procedures and associated software for problems from signal processing including computer aided techniques for the design and tuning of microwave devices. In particular, he is the author of the software toolboxes Dedale-HF and Presto-HF. 